\title{
One and Two Phonon Assisted Transitions between Exciton Spin States in a Quantum Dot
}

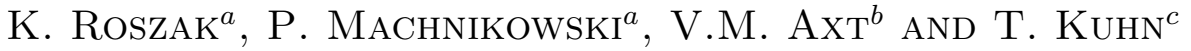 \\ ${ }^{a}$ Institute of Physics, Wrocław University of Technology \\ Wybrzeże Wyspiańskiego 27, 50-370 Wrocław, Poland \\ ${ }^{b}$ Theoretische Physik III, Universität Bayreuth, 95440 Bayreuth, Germany \\ ${ }^{c}$ Institut für Festkörpertheorie, Universität Münster \\ Wilhelm-Klemm-Str. 10, 48149 Münster, Germany
}

Exciton spin decay is studied in a self-assembled InAs/GaAs quantum dot. The spin relaxation results from an interplay of two factors: the BirPikus Hamiltonian and the short-range exchange interaction, leading to one and two phonon assisted transitions. We establish a hierarchy between the resulting transition rates and show the dominating role of transverse phonons for all the transitions.

PACS numbers: 72.25.Rb, 73.21.La, 78.67.Hc

\section{Introduction}

Interest in spin effects has been growing recently due to their possible applications in spin based electronics and as means of information storage in quantum computing. Consequently, relaxation and decoherence of confined spins are intensely researched. The case of an electron confined in a quantum dot (QD) has been thoroughly studied theoretically, including phonon-assisted processes $[1,2]$, while exciton spin has been experimentally investigated [3], but only some decay channels have been examined theoretically. Three relevant phonon-assisted processes have been identified so far. One is a transition to dark states due to carrier-phonon interactions and the spin-orbit coupling [4]. The other two include a transition between bright states [5] and a transition to dark states $[6,7]$ and are caused by the interplay of the Bir-Pikus Hamiltonian and the short-range exchange interaction.

In this paper, we study the processes resulting from the interplay of the Bir-Pikus Hamiltonian and the short-range exchange interaction. Apart from 
presenting a summary of the results concerning single-phonon assisted transitions [5-7], we concentrate on a new two-phonon assisted transition from bright to dark states. We compare the transition rates resulting from all three processes for typical GaAs/InAs self-assembled QD parameters. We also stress the dominating role of transverse phonons for all three processes.

\section{The system and the Hamiltonian}

The system under study consists of an exciton confined in a self-assembled QD in the presence of phonons. Eight exciton states are taken into account: four heavy hole (hh) and four light hole (lh) states (two bright and two dark states each). The Hamiltonian describing this system and its coupling to lattice modes may be written as $H=H_{\mathrm{QD}}+H_{\mathrm{sre}}+H_{\mathrm{ph}}+H_{\mathrm{BP}}$. The first term describes $\mathrm{QD}$ exciton energies,

$$
H_{\mathrm{QD}}=\sum_{n} E_{n}|n\rangle\langle n|,
$$

where the summation is taken over all states, $E_{n}=0$ for all hh exciton states and $E_{n}=E_{\mathrm{lh}}$ for all $\mathrm{lh}$ exciton states, where $E_{\mathrm{lh}}$ denotes the energy difference between hh and lh excitons. The second term accounts for the short-range exchange interaction [8],

$$
H_{\mathrm{sre}}=-\frac{2}{3} \Delta_{\mathrm{st}} \boldsymbol{J} \cdot \boldsymbol{\sigma}
$$

where $\Delta_{\text {st }}$ denotes the singlet-triplet splitting, $\boldsymbol{J}$ is the total hole angular momentum, and $\boldsymbol{\sigma}$ is the total electron angular momentum (in this case equal to the electron spin and represented by the Pauli matrices). The fourth term describes the free phonon evolution,

$$
H_{\mathrm{ph}}=\sum_{\boldsymbol{k}} \hbar \omega_{\boldsymbol{k}} b_{\boldsymbol{k}}^{\dagger} b_{\boldsymbol{k}}
$$

where $b_{\boldsymbol{k}}^{\dagger}, b_{\boldsymbol{k}}$ are phonon creation and annihilation operators, and $\omega_{\boldsymbol{k}}$ are the corresponding phonon frequencies. Throughout the paper, the phonon branch index will be implicit in $\boldsymbol{k}$ unless stated otherwise. The final term is the Bir-Pikus Hamiltonian $[8,9]$, which couples phonons to hole states,

$$
H_{\mathrm{BP}}=\left(a+\frac{5}{4} b\right) \varepsilon-b \sum_{i} \varepsilon_{i i} J_{i}^{2}-\frac{d}{2 \sqrt{3}} \sum_{i j} \varepsilon_{i j}\left[J_{i}, J_{j}\right]_{+},
$$

where $a, b$ and $d$ are deformation potential constants, $[\ldots, \ldots]_{+}$is an anticommutator, and $\varepsilon_{i j}$ are the strain tensor components, with $\varepsilon_{i j}=\varepsilon_{j i}$ and $\varepsilon=\varepsilon_{x x}+\varepsilon_{y y}+\varepsilon_{z z}$.

The states are labeled by a number denoting the projection of the total angular momentum and a letter $\mathrm{h}$ or $\mathrm{l}$, denoting hh and lh exciton states, respectively (a "+" or "-" sign denoting electron spin up or down is appended to distinguish between the two lh exciton dark states of total angular momentum 0). The basis is aligned as follows, $|2 \mathrm{~h}\rangle,|1 \mathrm{l}\rangle,|0 \mathrm{l}+\rangle,|-1 \mathrm{~h}\rangle,|1 \mathrm{~h}\rangle,|0 \mathrm{l}-\rangle,|-1 \mathrm{l}\rangle,|-2 \mathrm{~h}\rangle$, meaning 
that the first four states have electron spin up, the other four have electron spin down, and the projections of the hole angular momenta vary twice from $3 / 2$ to $-3 / 2$ going from left to right.

The explicit form of $H_{1}=H_{\mathrm{QD}}+H_{\mathrm{sre}}+H_{\mathrm{BP}}$ in the basis of all eight exciton states is

$$
H_{1}=\left(\begin{array}{cccccccc}
A_{-} & Q & P & 0 & 0 & 0 & 0 & 0 \\
Q^{*} & B_{-} & 0 & P & \frac{2 \mathrm{i} \Delta_{\mathrm{st}}}{\sqrt{3}} & 0 & 0 & 0 \\
P^{*} & 0 & B_{+} & -Q & 0 & \frac{4 \mathrm{i} \Delta_{\mathrm{st}}}{3} & 0 & 0 \\
0 & P^{*} & -Q^{*} & A_{+} & 0 & 0 & \frac{2 \mathrm{i} \Delta_{\mathrm{st}}}{\sqrt{3}} & 0 \\
0 & -\frac{2 \mathrm{i} \Delta_{\mathrm{st}}}{\sqrt{3}} & 0 & 0 & A_{+} & Q & P & 0 \\
0 & 0 & -\frac{4 \mathrm{i} \Delta_{\mathrm{st}}}{3} & 0 & Q^{*} & B_{+} & 0 & P \\
0 & 0 & 0 & -\frac{2 \mathrm{i} \Delta_{\mathrm{st}}}{\sqrt{3}} & P^{*} & 0 & B_{-} & -Q \\
0 & 0 & 0 & 0 & 0 & P^{*} & -Q^{*} & A_{+}
\end{array}\right),
$$

where

$$
A_{\mp}=\mp \Delta_{\mathrm{st}}+X, \quad B_{\mp}=E_{\mathrm{lh}} \mp \frac{\Delta_{\mathrm{st}}}{3}+Y .
$$

The singlet-triplet splitting parameter is $\Delta_{\mathrm{st}}=0.2 \mathrm{meV}$ and the heavy-light hole splitting is $E_{\mathrm{lh}}=40 \mathrm{meV}$. The operators $X, Y, P$ and $Q$ are given by

$$
\begin{aligned}
& X=\left(a+\frac{5}{4} b\right) \varepsilon-b \frac{3}{4}\left(\varepsilon_{x x}+\varepsilon_{y y}+3 \varepsilon_{z z}\right), \\
& Y=\left(a+\frac{5}{4} b\right) \varepsilon-b \frac{1}{4}\left(7 \varepsilon_{x x}+7 \varepsilon_{y y}+\varepsilon_{z z}\right), \\
& P=\frac{\sqrt{3}}{2} b\left(\varepsilon_{x x}-\varepsilon_{y y}\right)+\mathrm{i} d \varepsilon_{x y}, \\
& Q=-d\left(\varepsilon_{y z}+\mathrm{i} \varepsilon_{z x}\right) .
\end{aligned}
$$

The values of the deformation potential constants for GaAs are $b=2 \mathrm{eV}$ and $d=5 \mathrm{eV}$. The strain tensor components are defined by the formula

$$
\varepsilon_{i j}=\frac{1}{2}\left(\frac{\partial u_{i}}{\partial r_{j}}+\frac{\partial u_{j}}{\partial r_{i}}\right),
$$

where $\boldsymbol{u}(\boldsymbol{r})$ is the local displacement field. The displacement is quantized in terms of acoustic phonons,

$$
\boldsymbol{u}(\boldsymbol{r})=\mathrm{i} \sum_{\boldsymbol{k}} \sqrt{\frac{\hbar}{2 \rho V \omega_{\boldsymbol{k}}}} \hat{\boldsymbol{e}}_{\boldsymbol{k}}\left(b_{\boldsymbol{k}}+b_{-\boldsymbol{k}}^{\dagger}\right) \mathrm{e}^{\mathrm{i} \boldsymbol{k} \cdot \boldsymbol{r}},
$$

where $\hat{\boldsymbol{e}}_{\boldsymbol{k}}=-\hat{\boldsymbol{e}}_{-\boldsymbol{k}}$ are the real unit polarization vectors, $V$ is the crystal volume and 
$\rho=5360 \mathrm{~kg} / \mathrm{m}^{3}$ is the crystal density. Linear phonon dispersion is used with the speeds of longitudinal and transverse phonons $c_{\mathrm{l}}=5600 \mathrm{~m} / \mathrm{s}$ and $c_{\mathrm{t}}=2800 \mathrm{~m} / \mathrm{s}$, respectively.

Our goal is to investigate the relaxation of an initial bright hh exciton state. It is clear from the form of $H_{1}$ that bright hh exciton states are not coupled directly to any other hh exciton states. On the other hand, the energy difference between hh and $\mathrm{lh}$ exciton states, $E_{\mathrm{lh}}$, is large enough to make all transitions to light hole exciton states inefficient. However, in the second order, the interplay of short-range exchange interaction and the Bir-Pikus terms leads to a relaxation between bright hh exciton states, a simultaneous relaxation to dark hh exciton states, and a two-phonon assisted transition to dark states.

\section{Relaxation rates}

The Schrieffer-Wolff transformation [10] is used to calculate an effective Hamiltonian between two energetically close states which do not interact directly, but both interact with some third, energetically distant, state. This is done by performing a canonical transformation that eliminates the coupling between two of the states and applying it to the Hamiltonian responsible for the coupling between the other two.

In our system, lh exciton states are the energetically distant states, since the energy splitting between hh and lh exciton states is much larger than the splittings between hh exciton states. The elimination of the off-diagonal elements of short-range exchange interaction (that couple hh exciton states to lh exciton states of the same total angular momentum) and applying the respective transformation to the off-diagonal elements of the Bir-Pikus Hamiltonian (that couple hh exciton states to lh exciton states of different total angular momentum) leads to the effective interaction Hamiltonian of the form

$$
H_{\mathrm{int}}^{(1)}=\frac{2 \mathrm{i} \Delta_{\mathrm{st}}}{\sqrt{3} \widetilde{E}_{\mathrm{lh}}}\left(2 P^{*}|1 \mathrm{~h}\rangle\langle-1 \mathrm{~h}|+Q| 1 \mathrm{~h}\rangle\left\langle 2 \mathrm{~h}\left|+Q^{*}\right|-1 \mathrm{~h}\right\rangle\langle-2 \mathrm{~h}|\right)+\text { h.c. }
$$

where $\widetilde{E}_{\mathrm{lh}}=E_{\mathrm{lh}}-4 \Delta_{\mathrm{st}} / 3$. The first term of this Hamiltonian is responsible for the transition between bright hh exciton states, while the other two cause a transition to dark hh exciton states. We include also the fine structure splitting $\delta$ due to the long-range electron-hole exchange interaction.

The relaxation rates of the initial state $\left.|X\rangle=\frac{1}{\sqrt{2}}(|-1 \mathrm{~h}\rangle)+|1 \mathrm{~h}\rangle\right)$ resulting from this interaction Hamiltonian in the Fermi golden rule limit are plotted in Fig. 1 for different splittings between the bright states (fine structure splitting $\delta$ ). The details of the calculations may be found in Ref. [6]. Varying the fine structure splitting has little effect on the relaxation to dark states (left part), while it critically affects the relaxation between bright states (there is no transition between bright states for $\delta=0$; right part). The relaxation to dark states is over an order of magnitude faster than the transition to the other bright state; this is mainly due to the difference in energy splittings between the relevant states. For both processes the 
role of transverse phonons is dominating; in fact, the contribution of transverse phonons gives over $95 \%$ of the total relaxation rate for the transition to dark states, and over $97 \%$ for the transition between bright states.
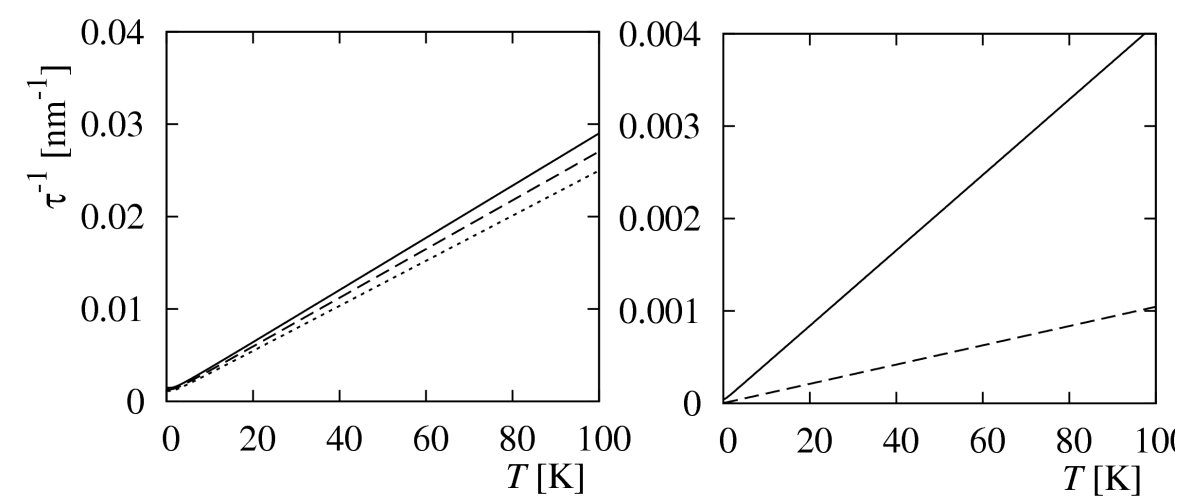

Fig. 1. Relaxation rate of the state $|X\rangle$ due to the transition to dark states (left part) and between bright states (right part). Solid line: $\delta=0.1 \mathrm{meV}$. Dashed line: $\delta=$ $0.05 \mathrm{meV}$. Dotted line: $\delta=0$.

Apart from the single-phonon transitions mediated by the interplay of the Bir-Pikus deformation Hamiltonian and short-range exchange, the Bir-Pikus Hamiltonian yields another transition channel. The off-diagonal elements of the Hamiltonian involving the operators $Q$ couple exciton states that differ by 1 in the total angular momentum, while the elements involving the operators $P$ describe the interaction between states differing in the total angular momentum by 2 . Therefore, both parts of the Hamiltonian couple hh exciton states to lh exciton states, but for a chosen lh exciton state two different hh exciton states, one dark and one bright, are coupled. The Schrieffer-Wolff transformation may be used again to find an effective Hamiltonian describing two-phonon assisted transitions between these bright and dark states.

Finding a transformation that eliminates one part of the Bir-Pikus Hamiltonian and applying it to the other part leads to the two-phonon effective interaction Hamiltonian of the form,

$$
H_{\mathrm{int}}^{(2)}=-\frac{\frac{8 \Delta_{\mathrm{st}}}{3} Q P}{E_{\mathrm{lh}}^{2}-\left(\frac{4 \Delta_{\mathrm{st}}}{3}\right)^{2}}(|-2 \mathrm{~h}\rangle\langle 1 \mathrm{~h}|+|-1 \mathrm{~h}\rangle\langle 2 \mathrm{~h}|)+\text { h.c. }
$$

This Hamiltonian describes a number of two-phonon processes, since $P$ and $Q$ contain both creation and annihilation operators.

It turns out that processes involving simultaneous phonon emission and absorption dominate over all other two-phonon assisted processes, since they allow phonons from a wide frequency range to contribute. The relaxation rate resulting from these transitions is of the form 


$$
\tau^{-1}=8 \pi \sum_{\boldsymbol{k q}, s}\left|p_{\boldsymbol{k}, s} q_{\boldsymbol{q}, s}+p_{\boldsymbol{q}, s} q_{\boldsymbol{k}, s}\right|^{2} n_{\boldsymbol{q}}\left(n_{\boldsymbol{k}}+1\right) \delta\left(\omega+\omega_{\boldsymbol{q}}-\omega_{\boldsymbol{k}}\right),
$$

with

$$
\begin{aligned}
p_{\boldsymbol{k}, s} & =\sqrt{\frac{\hbar k}{2 \rho c_{s}}} \mathcal{F}_{s}(\boldsymbol{k}) \frac{\sqrt{3}}{2} \mathrm{i} b\left(\begin{array}{c}
\cos ^{2} \theta\left(\cos ^{2} \phi-\sin ^{2} \phi\right) \\
-2 \cos \theta \cos \phi \sin \phi \\
\cos \theta \sin \theta\left(\cos ^{2} \phi-\sin ^{2} \phi\right)
\end{array}\right) \\
& -\sqrt{\frac{\hbar k}{2 \rho c_{s}}} \mathcal{F}_{s}(\boldsymbol{k}) \frac{d}{2}\left(\begin{array}{c}
2 \cos ^{2} \theta \cos \phi \sin \phi \\
\cos \theta\left(\cos ^{2} \phi-\sin ^{2} \phi\right) \\
2 \cos \theta \sin \theta \cos \phi \sin \phi
\end{array}\right), \\
q_{\boldsymbol{k}, s} & =\sqrt{\frac{\hbar k}{2 \rho c_{s}}} \mathcal{F}_{s}(\boldsymbol{k}) \frac{d}{2}\left(\begin{array}{c}
\cos \theta \sin \theta \\
\mathrm{i} \sin \theta \\
\cos ^{2} \theta-\sin { }^{2} \theta
\end{array}\right) \mathrm{e}^{-\mathrm{i} \phi},
\end{aligned}
$$

where $\boldsymbol{k}=[k \cos \theta \cos \phi, k \cos \theta \sin \phi, k \sin \theta]$, the form factors $\mathcal{F}_{s}(\boldsymbol{k})$ for Gaussian wave functions can be found in Ref. [11], and the three components correspond to the contributions of the longitudinal and two transverse acoustic phonon branches going from top to bottom.

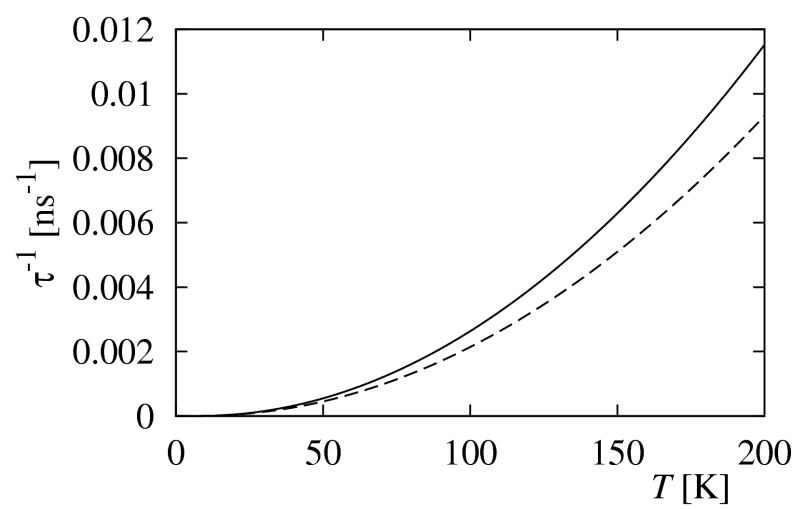

Fig. 2. Relaxation rate of the state $|X\rangle$ due to two-phonon effects for $\delta=0.1 \mathrm{meV}$ (solid line) and $\delta=0$ (dashed line).

The relaxation rates of an initial bright hh exciton state to dark states due to two-phonon assisted transitions are shown in Fig. 2 for two values of the fine structure splitting. They exhibit quadratic behavior as functions of temperature but, even up to room temperature, are small compared to single-phonon assisted transitions to dark states. For moderate temperatures and $\delta=0.1 \mathrm{meV}$, the two-phonon relaxation rate is comparable to the relaxation rate due to the single-phonon assisted transition between bright states. In contrast to the transition to 
bright states, the two-phonon assisted transition is weakly affected by the value of the fine structure splitting, and therefore is much larger for small values of $\delta$. Similarly to both single-phonon transitions, the two-phonon transition is dominated by transverse phonons which contribute over $97 \%$ of the relaxation rate.

\section{Conclusion}

We have studied spin transitions for a confined exciton, resulting from the interplay of the Bir-Pikus Hamiltonian and the short-range exchange interaction. We have shown that the single-phonon transition to dark states plays a dominating role for typical QD parameters. The two-phonon transition to dark states, which we also identified, is of the same order of magnitude as the single-phonon transition to bright states for moderate temperatures and fine structure splittings. At low temperatures the two-phonon transition becomes negligible, while the transition between bright states vanishes with vanishing fine structure splitting. For all three transitions the contribution of transverse acoustic phonons constitutes over $95 \%$ of the relaxation rate.

\section{References}

[1] V.N. Golovach, A.V. Khaetskii, D. Loss, Phys. Rev. Lett. 93, 016601 (2004).

[2] A.V. Khaetskii, Y.V. Nazarov, Phys. Rev. B 64, 125316 (2000).

[3] K.C. Hall, E.J. Koerperick, T.F. Boggess, O.B. Shchekin, D.G. Deppe, Appl. Phys. Lett. 90, 053109 (2007).

[4] E. Tsitsishvili, R. v. Baltz, H. Kalt, Phys. Rev. B 72, 155333 (2005).

[5] E. Tsitsishvili, R. v. Baltz, H. Kalt, Phys. Rev. B 67, 205330 (2003).

[6] K. Roszak, V.M. Axt, T. Kuhn, P. Machnikowski, Phys. Rev. B 76, 195324 (2007), see also Erratum, submitted for publication.

[7] E. Tsitsishvili, R. v. Baltz, H. Kalt, Phys. Status Solidi C 1, 3 (2004).

[8] G.L. Bir, G.E. Pikus, Symmetry and Strain-Induced Effects in Semiconductors, Wiley, New York 1974.

[9] L.M. Woods, T.L. Reinecke, R. Kotlyar, Phys. Rev. B 69, 125330 (2004).

[10] J.R. Schrieffer, P.A. Wolff, Phys. Rev. 149, 491 (1966).

[11] A. Grodecka, L. Jacak, P. Machnikowski, K. Roszak, in: Quantum Dots: Research Developments, Ed. P.A. Ling, Nova Science, New York 2005. 